\title{
Early Effects of 2-Acetylaminofluorene and N-Hydroxy-2- Acetylaminofluorene on Mitochondrial Outer Membrane Enzyme Activities of Rat Liver
}

\author{
Takemi YOSHIDA, Toshinori YAMAMOTO, Yoichi MATSUURA* \\ and Yukio KUROIWA \\ Department of Biochemical Toxicology, School of Pharmaceutical Sciences, \\ Showa University, 1-5-8 Hatanodai. Shinagawa-ku. Tokyo 142, Japan \\ *Department of Hygienic Chemistry. Showa College of Pharmaceutical Sciences, \\ 5-1-8 Tsurumaki, Setagaya-ku, Tokyo 154. Japan
}

Accepted August 21, 1985

\begin{abstract}
During the early stages of the carcinogen 2-acetylaminofluorene (2AAF) feeding, there were marked decreases of hepatic mitochondrial type $B$ monoamine oxidase (MAO) and kynurenine 3-hydroxylase activities in male rats, but not in female rats. The administration of $\mathrm{N}$-hydroxy-2-AAF (N-OH-2-AAF), a proximate carcinogenic metabolite of 2 -AAF, to male rats also resulted in the decrease of both enzyme activities. The findings indicate that type B MAO and kynurenine 3-hydroxylase which reside in the outer mitochondrial membranes are susceptible to 2-AAF during the early stages of its hepatocarcinogenesis.
\end{abstract}

We have recently shown that there was a decrease of mitochondrial MAO activity in the liver of rats fed a diet containing 2 -AAF (1). The decrease of MAO activity following the exposure of rats to dietary 2-AAF was preferential to type B MAO. Type A MAO activity was not changed by such treatment of rats with 2-AAF. In addition, a sex-related effect of 2-AAF on MAO was also seen in rats, MAO activity in male rats being significantly decreased but to a less extent or not at all in female rats during the early stages, in accordance with its hepatocarcinogenic ability in both sexes $(2,3)$.

It has shown that the outer mitochondrial enzyme activities such as kynurenine 3hydroxylase, rotenone-insensitive NADH cytochrome $c$ reductase in addition to MAO are susceptibly reduced by some physiological changes, especialiy in riboflavin deficiency $(4,5)$, hyperthyroidism induced by thyroxine administration $(6,7)$ and liver regeneration after partial hepatectomy (8). These findings prompted us to examine whether 2-AAF produces any changes in the activities of the outer mitochondrial kyn- urenine 3-hydroxylase, rotenone-insensitive $\mathrm{NADH}$ cytochorme $c$ reductase in addition to type $B$ MAO, and whether the effect of the carcinogen on the enzyme activities has any similarities to those of physiologicallyinduced decreases. Additionally, to examine further a possible correlation between the effect of 2-AAF on the enzyme activities and its hepatocarcinogenesis, the present investigation also dealt with the effect of $\mathrm{N}$ $\mathrm{OH}-2-\mathrm{AAF}$ on the outer mitochondrial enzyme activities.

\section{Materials and Methods}

Chemicals: 2-AAF was obtained from Tokyo Kasei Co. 2-Nitrofluorene (2-NF) was purchased from Aldrich Chemical Co. N$\mathrm{OH}-2-\mathrm{AAF}$ was prepared from 2-NF as described by Miller et al. (9). All other chemicals were of the highest grade available commercially.

Animals and treatment: Male and female Donryu rats, weighing initially $120 \mathrm{~g}$ to $130 \mathrm{~g}$ (4 weeks of age), were fed a commercially available basal diet for one week. Thereafter, the rats were divided into two groups; one 
group of rats was fed a basal diet, and the other group was fed a basal diet containing $0.05 \%$ 2-AAF. In some experiments, an equimolar amount of 2-AAF and $\mathrm{N}-\mathrm{OH}-2$ AAF dissolved in corn oil was administered to male rats daily for seven days.

Preparation of mitochondria: Rats were sacrificed at the times indicated in Fig. and Tables. The liver was perfused in situ with cold $0.09 \% \mathrm{NaCl}$ solution, excised, weighed and homogenized with 9 vol. of $0.25 \mathrm{M}$ sucrose solution. The mitochondrial fraction from the homogenate was prepared as described previously (1).

Enzyme assays: Type B MAO activity was determined spectrophotometrically by the method of Tabor et al. (10) by using benzylamine as the substrate. Kynurenine 3hydroxylase activity was assayed as described by Stevens and Henderson (11). Rotenoneinsensitive NADH cytochrome $c$ reductase activity was determined according to the method of Sottocasa et al. (12). Mitochondrial protein concentration was determined by the method of Lowry et al. (13) by using bovine serum albumin as the standard.

\section{Results}

Table 1 shows the effect of 2-AAF feeding on mitochondrial type $\mathrm{B} M A O$, kynurenine 3-hydroxylase and rotenone-insensitive NADH cytochrome $c$ reductase activities in the livers of male and female rats. In accordance with the decrease of type $B$ MAO activity as reported previously (1), there was a marked decrease of kynurenine 3-hydroxylase activity when determined one week after the exposure of male rats to a 2-AAFcontaining diet, but rotenone-insensitive $\mathrm{NADH}$ cytochrome $c$ reductase activity was not changed under the experimental conditions. It was also found that these three enzyme activities were not changed appreciably in female rats exposed to $2-A A F$. The results support the previous findings (1) and add further evidence that there is a sexrelated difference in the response to 2-AAF with respect to the decrease of mitochondrial enzyme activities including MAO.

Since 2-AAF feeding resulted in the decrease of kynurenine 3-hydroxylase activity of male rat liver, we also carried out the time course study on the effect of the carcinogen feeding on the enzyme activity, as shown in Fig. 1. Concomitant with the decrease of type B MAO activity as reported previously (1), there was a marked decrease of kynurenine 3-hydroxylase activity during 2-AAF feeding as compared to the controls at each time period. The decrease of kynurenine 3hydroxylase activity of about $70 \%$ of the controls appeared within one week following the initial exposure of male rats to dietary 2-AAF. The decreased kynurenine 3hydroxylase activity which reached minimum at three weeks after the initiation of 2-AAF feeding was sustained for five weeks, as so far examined. Additionally, the decreased activity of kynurenine 3-hydroxylase did not return to control levels by two weeks when

Table 1. Effect of 2-AAF feeding on mitochondrial type B MAO. kynurenine 3-hydroxylase and rotenoneinsensitive $\mathrm{NADH}$ cytochrome $c$ reductase activity of male and female rat liver

\begin{tabular}{|c|c|c|c|}
\hline & $\begin{array}{c}\text { Type B MAO } \\
(\mathrm{nmol} / \mathrm{mg} \text { protein } / 30 \mathrm{~min})\end{array}$ & $\begin{array}{c}\text { Kynurenine } \\
\text { 3-hydroxylase } \\
\text { (nmol/mg protein/min) }\end{array}$ & $\begin{array}{l}\text { Rotenone-insensitive } \\
\text { NADH cytochrome } c \\
\text { reductase } \\
\text { (nmol/mg protein/min) }\end{array}$ \\
\hline \multicolumn{4}{|l|}{ Male rats } \\
\hline Control & $206.8 \pm 13.4$ & $3.35 \pm 0.11$ & $334.1 \pm 6.9$ \\
\hline 2 -AAF-fed & $142.6 \pm 3.3^{*}$ & $2.08 \pm 0.30^{*}$ & $319.4 \pm 14.2$ \\
\hline \multicolumn{4}{|l|}{ Female rats } \\
\hline Control & $163.5 \pm 7.4$ & $4.09 \pm 0.04$ & $323.4 \pm 24.6$ \\
\hline 2-AAF-fed & $156.5 \pm 3.9$ & $3.88 \pm 0.05$ & $297.3 \pm 22.7$ \\
\hline
\end{tabular}

Male and female rats were fed a diet containing $0.05 \% 2$-AAF and sacrificed at seven days after the initiation of the carcinogen feeding. Each value is the mean $\pm S$. E. of three or four rats. "Significantly different from the control $(P<0.05)$ 
the diet was switched to the basal diet at one or three weeks after the exposure of rats to dietary 2-AAF.

Finally, experiments were carried out to examine whether $\mathrm{N}-\mathrm{OH}-2-\mathrm{AAF}$, a proximate carcinogenic metabolite of 2-AAF $(9,14-16)$. also has a decreasing effect on these mitochondrial enzyme activities. As shown in Table 2, the repetitive administration of either 2-AAF or $\mathrm{N}$-OH-2-AAF resulted in a significant decrease of type $B$ MAO and kynurenine 3-hydroxylase activities. However, the extent of the decrease of type B MAO and kynurenine 3 -hydroxylase activities produced

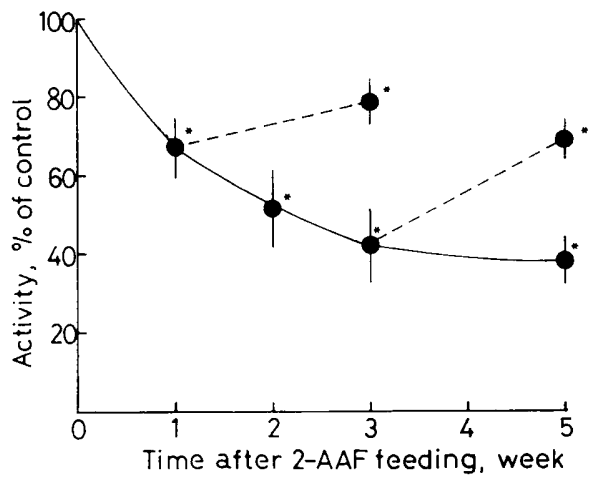

Fig. 1. Time course of the effect of 2-AAF feeding on mitochondrial kynurenine 3-hydroxylase activity of male rat liver. Rats were fed a diet containing $0.05 \% 2-A A F$ and sacrificed at the times indicated. The values, expressed as percentage of the corresponding control at each time period, are the mean \pm S.E. of three or four rats. A dotted line indicates that rats were fed the basal diet following 2-AAF feeding. The control values ranged from 2.51 to $3.54 \mathrm{nmol} / \mathrm{mg}$ protein/min during the time course study. *Significantly different from the control $(P<0.05)$. by the administration of $\mathrm{N}-\mathrm{OH}-2$-AAF was almost similar to that seen in 2-AAF administration despite that the dose administered to rats was same.

\section{Discussion}

This investigation has revealed that the carcinogen 2-AAF produced the decrease of mitochondrial type B MAO and kynurenine 3-hydroxylase activities, but not rotenoneinsensitive $\mathrm{NADH}$ cytochrome $c$ reductase activity, during the early stages of its feeding. Sex-related difference in the response to 2AAF feeding has also been shown in this study, male rats showing decreases in both type B MAO and kynurenine 3-hydroxylase activities following the carcinogen feeding, but not female rats (Table 1). Additionally, the decreased pattern of kynurenine 3hydroxylase activity after the initiation of 2 AAF feeding (Fig. 1) was almost similar to that seen in case of the decrease of type $B$ MIAO activity as reported previously (1). It should be also pointed out that $\mathrm{N}-\mathrm{OH}-2$-AAF, a proximate carcinogenic metabolite of 2 AAF (9, 14-16), produced the decrease of type B MAO and kynurenine 3-hydroxylase activities after the repetitive administration to male rats (Table 2 ). This finding may suggest that the observed decrease of type $B$ MAO and kynurenine 3-hydroxylase activities following 2-AAF feeding could be mediated, at least in part, through the formation of $\mathrm{N}$ $\mathrm{OH}-2$-AAF. However, both 2-AAF and $\mathrm{N}$ $\mathrm{OH}$-2-AAF may not decrease type $\mathrm{B}$ MAO and kynurenine 3-hydroxylase activities by binding to or modifying the enzyme protein, because both carcinogens inhibited the former enzyme activity at rather higher con-

Table 2. Effects of 2-AAF and N-OH-2-AAF on mitochondrial type B MAO and kynurenine 3hydroxylase activity of male rat liver

\begin{tabular}{lcc}
\hline Treatment & $\begin{array}{c}\text { Type B MAO } \\
(\mathrm{nmol} / \mathrm{mg} \text { protein/30 } \mathrm{min})\end{array}$ & $\begin{array}{c}\text { Kynurenine } \\
3 \text {-hydroxylase } \\
(\mathrm{nmol} / \mathrm{mg} \text { protein } / \mathrm{min})\end{array}$ \\
\hline Control & $214.3 \pm 2.2$ & $2.69 \pm 0.22$ \\
2-AAF & $141.2 \pm 12.8^{*}$ & $1.13 \pm 0.02^{*}$ \\
N-OH-2-AAF & $149.8 \pm 3.5^{*}$ & $1.24 \pm 0.10^{*}$ \\
\hline
\end{tabular}

2-AAF or N-OH-2-AAF was administered orally to rats at a dose of $200 \mu \mathrm{mol} / \mathrm{kg}$ daily for seven days and sacrificed $24 \mathrm{hr}$ after the last administration. Values are the mean \pm S.E. of three or four rats. ${ }^{*}$ Significantly different from the ccntrol $(P<0.05)$. 
centrations (approximately $1 \times 10^{-4} \mathrm{M}$ for $50 \%$ inhibition) and did not inhibit kynurenine 3-hydroxylase activity when added in vitro to the reaction mixture (data not shown).

The observed difference in response to 2$A A F$ between male and female rats with respect to the decrease of type $B$ MAO and kynurenine 3-hydroxylase activities may be not due to the difference in the extent of the formation of N-OH-2-AAF, because both male and female rats have abilities to form $\mathrm{N}$-OH-2-AAF to a similar extent as reported by Malejka-Giganti et al. (17) and MalejkaGiganti and Ritter (18). It has shown that male rats have a greater $\mathrm{N}-\mathrm{OH}-2-\mathrm{AAF}$ sulfotransferase activity than female rats, corresponding well to its hepatocarcinogenic ability (16). Although it remains to be elucidated whether it is necessary for $\mathrm{N}-\mathrm{OH}$ 2-AAF to be further esterified to exert its decreasing effect on type B MAO and kynurenine 3-hydroxylase activities; if this were the case, the observed sex-difference in response to 2 -AAF could be well explained on the basis of the difference in sulfotransferase activity. We found higher $\mathrm{N}-\mathrm{OH}-2$ AAF sulfotransferase activity in male Donryu rat liver than the female ones used in this study ( $T$. Yoshida et al., unpublished results); however, further in-depth study will be required to clarify the observed sex-difference from this aspect.

With respect to the effect of the hepatocarcinogens on mitochondrial enzymes, Kizer and Howell (19) have shown that there was a decrease or loss of kynurenine 3hydroxylase activity during $3^{\prime}$-methyl-4dimethylaminoazobenzene (3'-Me-DAB) feeding or the carcinogen-induced hepatomas respectively. In addition, type B MAO activity was also decreased during the early stages of 3'-Me-DAB feeding (20). Considering these facts, it would be reasonable to point out that mitochondrial type $B$ MAO and kynurenine 3-hydroxylase are susceptibly decreased by 2-AAF and 3'-Me-DAB during the early stages of their hepatocarcinogenesis.

Although it remains to be elucidated how 2-AAF and $\mathrm{N}-\mathrm{OH}-2$-AAF reduce type $\mathrm{B}$ $\mathrm{MAO}$ and kynurenine 3-hydroxylase activities the decreased patterns of the enzyme activities are similar to that occurring during liver regeneration after partial hepatectomy (8). Thus, further studies will be also necessary to define whether the decrease of type $\mathrm{B}$ MAO and kynurenine 3-hydroxylase activities by 2 -AAF feeding is a phenomenon accompanying the entire course of hepatocarcinogenesis by this agent, including its toxic effect on liver cells.

Acknowledgement: The authors wish to thank Mr. Kei-ichi Seki and Miss Nobuko Tei for their expert technical assistances in this study.

\section{References}

1 Yoshida, T., Uchida, A., Yamamoto, T. and Kuroiwa, Y.: Early changes in mitochondrial monoamine oxidase activity of rat liver during 2 -acetylaminofluorene feeding. Biochim. Biophys. Acta 677, 280-286 (1981)

2 Skoryna, S.C. and Webster, D.R.: Production of cirrhosis and liver tumors in male rats using high dose of 2-acetylaminofluorene. Proc. Soc. Exp. Biol. Med. 78, 62-67 (1951)

3 Teebor, G.W. and Seidman, I.: Retention of metabolic regulation in the hyperplastic nodule induced by $\mathrm{N}$-2-fluorenylacetamide. Cancer Res. 30, 1095-1101 (1970)

4 Hawkins, J.: Amine oxidase activity of rat liver in riboflavin deficiency. Biochem. J. 51, 399404 (1951)

5 Kwatra, M.M. and Sourkes, T.L.: Monoamine oxidase $A$ and $B$ activities in liver of riboflavindeficient rats. Biochem. Pharmacol. 29, 26932694 (1980)

6 Okamoto, $H_{\text {.: }}$ Influence of $L$-thyroxine on kynurenine 3-hydroxylase, monoamine oxidase, and rotenone-insensitive NADH cytochrome $\mathrm{c}$ reductase in mitochondrial outer membrane. Biochem. Biophys. Res. Commun. 43, 827-833 (1971)

7 Tong, J.H. and D'lorio, A.: Differential effects of L-thyroxine on cardiac and hepatic monoamine oxidase activity toward benzylamine and serotonin. Endocrinology 98, 761-766 (1976)

8 Gear, A.R.L.: Inner- and outer-membrane enzymes of mitochondria during liver regeneration. Biochem. J. 120, 577-587 (1970)

9 Miller, E.C., Miller, J.A. and Hartman, H.A.: NHydroxyacetylaminofluorene: A metabolite of 2 -acetylaminofluorene with increased carcinogenic activity in the rat. Cancer Res. 21, 815824 (1961)

10 Tabor, C.W., Tabor, H. and Rosenthal, S.M.: Purification of amine oxidase from beef plasma. 
J. Biol. Chem. 208, 645-661 (1954)

11 Stevens, C.O. and Henderson, L.M.: Riboflavin and hepatic kynurenine 3-hydroxylase. J. Biol. Chem. 234, 1191-1194 (1959)

12 Sottocasa, G.L., Kuylenstierna, B., Ernster, L. and Bergstrand, A.: An electron-transport system associated with the outer membrane of liver mitochondria. J. Cell Biol. 32, 415-438 (1967)

13 Lowry, O.H., Rosebrough, N.J., Farr, A.L. and Randall, R.J.: Protein measurement with the Folin phenol reagent. J. Biol. Chem. 193, 265275 (1951)

14 Miller, E.C., Lotlikar, P.D., Pitot, H.C., Fletcher, T.L. and Miller, J.A.: N-Hydroxy metabolites of 2-acetylaminofluorene and 7-fluoro-2-acetylaminofluorene as proximate carcinogens in the rat. Cancer Res. 26, 2239-2247 (1966)

15 Miller, E.C., Mil!er, J.A. and Enomoto, M.: The comparative carcinogenicities of 2 -acetylaminofluorene and its $\mathrm{N}$-hydroxy metabolite in mice, hamsters and guinea pigs. Cancer Res. 24,
2018-2032 (1964)

16 Miller, J.A.: Carcinogenesis by chemicals: An overview. Cancer Res. 30, 559-576 (1970)

17 Malejka-Giganti, D., Mclver, R.C., Glasebrook, A.L. and Gutmann, H.R.: Induction of microsomal N-hydroxylation of $\mathrm{N}$-2-fluorenylacetamide in rat liver. Biochem. Pharmacol. 27, 6169 (1978)

18 Malejka-Giganti, D. and Ritter, C.L.: Effects of acute and chronic treatment of rats with $\mathrm{N}-2$ fluorenylacetamide on its $\mathrm{N}$-hydroxylation and on hemoproteins of hepatic microsomes. Res. Commun. Chem. Pathol. 28, 53-64 (1980)

19 Kizer, R.E. and Howell, B.A.: The detection of kynurenine hydroxylase activity during hepatocarcinogenesis. JNCl 30, 675-686 (1963)

20 Egashira, T., Uchida, A. and Kuroiwa, Y.: Monoamine oxidase activity in liver of rats given 3'-methyl-4-dimethylaminoazobenzene (3'-Me$D A B)$ in the diet. Biochem. Pharmacol. 28, $1561-1563$ (1979) 